\title{
Publisher Correction: Structure and assembly of calcium homeostasis modulator proteins
}

Johanna L. Syrjanen, Kevin Michalski, Tsung-Han Chou (D), Timothy Grant, Shanlin Rao, Noriko Simorowski, Stephen J. Tucker (D), Nikolaus Grigorieff (D) and Hiro Furukawa $\mathbb{D}$

Correction to: Nature Structural and Molecular Biology https://doi.org/10.1038/s41594-019-0369-9, published online 27 January 2020.

In the version of this article initially published, in Fig. 4a, the eye on the left was incorrectly labeled "Panel d" instead of "Panel c". The eye on the right was incorrectly labeled "Panel c" instead of "Panel b". The errors have been corrected in the HTML and PDF versions of the article.

Published online: 17 February 2020

https://doi.org/10.1038/s41594-020-0396-6

(C) The Author(s), under exclusive licence to Springer Nature America, Inc. 2020 